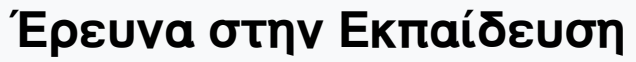

Tóp. 5, Ap. 1 (2016)

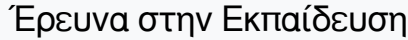

$\operatorname{son} 2241700$

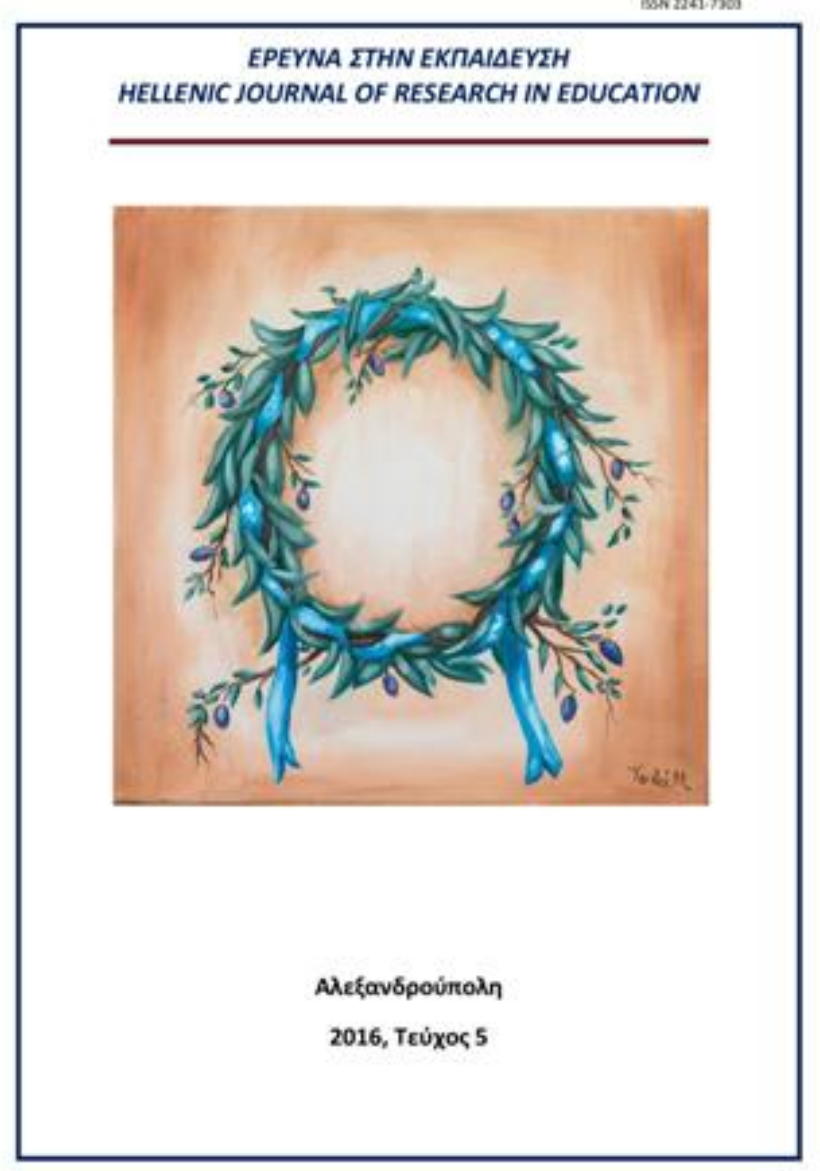

Apprentissages et professionnalité au sein des structures d'accueil des jeunes enfants

Gilles Brougère

doi: $10.12681 /$ hire.10825

Copyright @ 2016, Gilles Gilles Brougère

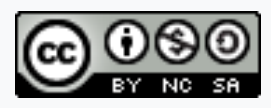

Aঠ¿ıа Xpńбnৎ Creative Commons Attribution-NonCommercial-ShareAlike 4.0.

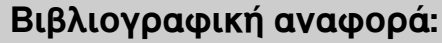

Brougère, G. (2016). Apprentissages et professionnalité au sein des structures d'accueil des jeunes enfants. 'E $\rho \varepsilon u v a$

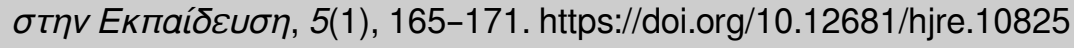




\title{
Apprentissages et professionnalité au sein des structures d'accueil des jeunes enfants
}

\author{
Gilles Brougère \\ Experice, Université Paris 13 - Sorbonne Paris Cité
}

\section{Résumé}

Cet article vise à poser la question d'une professionnalisation du personnel des structures d'éducation et d'accueil de la petite enfance qui ne soit pas pensée en opposition avec les connaissances et pratiques parentales. En ce qui concerne le soin et l'éducation des enfants nous supposons une continuité qui renvoie à l'importance de la culture et des apprentissages informels. Mais les professionnels acquièrent d'autres compétences concernant leurs relations avec les adultes (parents et collègues) et l'articulation entre la singularité de chaque enfant et l'organisation d'une vie collective. ${ }^{1}$

\begin{abstract}
The aim of this article is to interrogate the professionalization of the Early Childhood education and care staff. We don't consider it as the opposite of parent's knowledge and practice. There is a continuity which refers to the importance of culture and informal learning. The staff achieves other competencies concerning the relationships with adults (parents and workmate) and the connexion between singularity of each child and organisation of the community.
\end{abstract}

(C) 2016, Gilles Brougère

A $\delta \varepsilon 1 \alpha$ CC-BY-SA 4.0

Mots clés: Accueil et éducation de la petite enfance, professionnalisation, apprentissage informel, répertoires de pratiques

Key words: Early childhood education and care, professionalization, informal learning, repertoires of practices

Si l'on s'intéresse à la question des professionnelles du préscolaire et tout particulièrement de l'accueil des moins de 3 ans, on perçoit une tension entre deux visions de la professionnalité. D'une part certains considèrent qu'il n'y a pas besoin d'être professionnelle pour s'occuper des enfants, tout le monde pouvant le faire ou tout au moins toute personne ayant l'expérience maternelle, ce qui conduit à dévaloriser la profession, voire à la sous-payer. D'autre part la position la plus commune aujourd'hui

\footnotetext{
${ }^{1}$ Une première version de ce texte a fait l'objet d'une communication invitée au colloque « Early Childhood Education and Care : New trends », Lebanese University, Beyrouth, 28-29 novembre 2014.
} 
est de considérer que seule une professionnelle peut s'occuper des enfants, ce qui revient à dévaloriser l'expérience parentale avec laquelle il faudrait rompre pour devenir professionnelle; cela peut conduire à mettre sous tutelle les parents.

Cette tension renvoie à différentes questions entrelacées :

- La question de la continuité ou de la discontinuité entre les soins (le care) domestiques, dans l'espace familial et les soins professionnalisés dans des structures collectives d'accueil. Le problème est qu'il y à la fois continuité et discontinuité. Nous ne sommes pas en face de deux pôles qui s'opposeraient, du fait de l'existence de situations intermédiaires comme la garde alternative et réciproque par des mères de leurs enfants avec d'autres, la garde rémunérée ou non par un membre de la famille, par une personne à domicile, par une personne qui dans son cadre familial accueille des enfants (les assistantes maternelles en France).

- La question du rapport entre care et rémunération, et donc professionnalisation : le care comme activité gratuite, plutôt féminine est ancrée dans l'histoire, renvoie à la logique du don, de la vocation (genrée ${ }^{2}$, féminine) et a pu se heurter à une logique professionnelle ; ces questions sont aujourd'hui largement développées dans la littérature (Tronto, 2009).

- L'articulation entre le care, le soin et l'éducation, le premier renvoyant plus à la logique maternelle, le second impliquant une éventuelle technicisation. S 'agit-il de garder ou d'éduquer des enfants? Mais là encore il faut considérer que toute garde a une dimension éducative (inévitable) et que tout système éducatif remplit une fonction de garde y compris bien au-delà de l'éducation préscolaire.

- L'articulation entre le rôle des parents comme premiers éducateurs et celui d'intervenants extérieurs à la famille, qui peuvent porter d'autres valeurs, d'autres visions éducatives

Comment sortir de ces dilemmes sans dévaloriser les professionnelles ou les parents ? Cela suppose une analyse de l'éducation préscolaire, des structures d'accueil et de la profession qui peut être en rupture avec les visions les plus simples.

En effet la vision la plus répandue est celle qui conduit à considérer que la profession d'éducateur ou d'enseignant de la petite enfance s'appuie sur une compétence technique liée à la maîtrise d'un savoir particulier, celui du développement de l'enfant. Elle conduit à relier directement ce savoir et la pratique éducative, ce qui conduit à définir de bonnes pratiques universelles. Mon approche est plutôt liée à l'idée que l'éducation s'enracine dans la culture, les valeurs locales, ce qui met à distance l'idée du lien direct entre psychologie et pratique. C'est affaire de pédagogie, de choix quant à l'enfant que l'on souhaite, d'insertion dans une société ou de projets de changement de celle-ci. On ne peut tirer de la psychologie une pédagogie, celle-ci est toujours un choix sur les valeurs

Il faut donc rompre avec cette vision trop simple; cela ne signifie pas qu'un savoir psychologique parmi d'autres savoirs possibles ne puisse être mobilisé dans la pratique éducative, mais simplement que la pratique ne peut être simplement et directement issue de celui-ci. Les connaissances psychologiques doivent être mises en relation avec le contexte culturel et local dans une logique non pas de production d'une norme mais de soutien à l'interprétation des comportements. Il faut plutôt voir dans la psychologie un outil pour l'analyse qu'une norme pour l'action. C'est à partir de l'analyse de la situation et des comportements qu'une action pédagogique dans un contexte spécifique tenant compte des valeurs culturelles peut être engagée.

\footnotetext{
${ }^{2}$ «Genrée » est entendu ici comme marquée par la différence sociale entre les sexes.
} 


\section{Redéfinir la professionnalité}

Une première voie consiste à redéfinir la professionnalité qui n'est pas s'occuper des enfants mais gérer ou intervenir dans un collectif de parents, d'enfants et de professionnelles, donc gérer des relations avec des adultes. Les soins aux enfants doivent être redéfinis dans ce cadre sans nécessairement rompre avec l'expérience parentale.

En effet l'erreur est souvent de ne pas voir où se situe la véritable professionnalité qui est moins s'occuper d'un enfant que s'occuper d'un enfant qui n'est pas le sien et avec lequel il n'existe pas de lien familial qui justifie la relation comme c'est le cas des parents ou des grands-parents, voire d'autres membres de la famille.

Cela suppose que la professionnelle doit gérer (et apprendre à gérer):

\section{a. La relation avec les parents, leurs angoisses, leurs inquiétudes, leurs questions}

Accueillir les parents, les rassurer, leur donner à voir ce qui se passe (documenter la vie de l'enfant), mais aussi connaitre mieux l'enfant pour adapter la structure collective aux besoins individuels. Nous avons pu étudier cela (Brougère, Roucous, Chanu, 2001) dans le cas d'assistantes maternelles en France dont l'apprentissage «sur le tas », c'est-à-dire en cours d'activité professionnelle, consiste à mieux gérer la relation avec les parents. Ainsi peuvent-elles utiliser le jeu et leur connaissance du jeu de l'enfant acquise en particulier par la fréquentation de ludothèques comme thème de discussion pour ne pas se limiter à une vision étroite des soins ni tomber dans une logique éducative formelle. Les parents apparaissent très intéressés par une meilleure connaissance des activités de jeu de leurs enfants et peuvent ainsi demander à l'assistante maternelle des conseils pour l'achat de jeux et jouets. Elles développent une professionnalité reconnue en devenant capables de gérer la relation avec les parents et dans le même temps valoriser leur travail qui ne se limite pas aux soins en montrant comment elles sont attentives à observer et enrichir le jeu des enfants qu'elles gardent.

Une autre étude (Brougère, Moreau, 2014) a étudié des crèches parentales qui ne distinguent pas ou peu l'activité des parents et des éducateurs auprès des enfants, mais le professionnel est là pour que parents et professionnels travaillent ensemble. Il permet de faire exister une collectivité où tous, avec leurs diversités nombreuses dans ces crèches fréquentées par des familles issues de cultures différentes, peuvent travailler ensemble sans mettre de côté ses façons de faire, de s'occuper des enfants.

Une dernière étude (Rupin, 2014) a montré comment des éducatrices de jardins d'enfants chiliens arrivent à mobiliser des parents pour les impliquer dans la vie de la structure. La spécificité de leur action est celle de mobiliser la communauté, les différents acteurs pour enrichir l'environnement éducatif des enfants, elles-mêmes n'étant que peu formées aux techniques pédagogiques.

\section{b. La relation avec les autres professionnelles}

La professionnelle dans nombre de situations (qui justement se distinguent de la famille et des structures de type familial comme l'assistante maternelle en France) n'est pas seule et la coordination entre professionnelles (aux mêmes tâches ou à des tâches différentes) est essentielle. Il y a un besoin de coordination et de coopération - passer le relais, travailler ensemble - qui définit la logique professionnelle par rapport à la logique parentale sans dévaloriser celle-ci. Ce sont simplement les 
conditions qui sont différentes. Ce qui diffère c'est moins la relation entre adulte et enfants, que le fait de développer cette relation à travers plusieurs adultes aux tâches ou aux horaires bien définis. Il s'agit de faire de ce qui est trop souvent vu comme un défaut (le fait que l'enfant est confronté à différentes personnes), une richesse, la possibilité de nouer des relations avec des adultes différents qui travaillent ensemble de façon cohérente, mais chacun tel qu'il est, avec sa personnalité, c'est-à-dire en aucun cas de façon identique. Si la structure préscolaire doit tenir compte de la singularité de chaque enfant et non le considérer comme interchangeable, de façon symétrique, elle doit construire une collectivité avec des professionnelles singulières capables de coopérer sans se fondre dans un moule. La prise en compte de la singularité des adultes devient un moyen de préserver celle des enfants.

\section{c. Le fait qu'il s'agit d'un collectif d'enfants}

Ce qui caractérise ce collectif est la présence d'enfants du même âge (par opposition à une famille nombreuse qui suppose des âges plus ou moins échelonnés). Cela implique une redéfinition, une modification des tâches pour les rendre compatibles avec le nombre. Un exemple parfois douloureux (cela peut se passer assez mal y compris dans des structures excellentes par ailleurs) est le repas, marqué par le nombre d'enfant, l'attente, les conditions matérielles. L'enjeu professionnel est bien là, dans l'articulation entre la singularité de chaque enfant (qui peut tout particulièrement être prise en compte dans la famille) et le nombre qui implique des contraintes avec l'idée que les premiers modèles de fonctionnement (un peu militaires comme le fut la salle d'asile) ne sont plus acceptables. C'est sans doute le défi des structures collectives qui conduit parfois à préférer de petites structures, ou des structures divisées en sections de taille réduite ou encore le mélange des âges pour limiter le nombre des enfants du même âge. C'est sans doute là l'enjeu le plus difficile, concilier le respect des personnes (enfants comme professionnelles) avec un fonctionnement nécessairement collectif. C'est là qu'il faut trouver des pratiques qui pour beaucoup renvoient plus à des logiques culturelles d'organisation qu'à des normes issues de la psychologie.

Ces façons de saisir la professionnalité ont l'avantage de ne pas dévaloriser le parent dont on ne nie pas la compétence quant au traitement d'un enfant singulier, le professionnel n'ayant pas nécessairement de compétences supérieures à ce niveau (ou s'il a une compétence il peut la devoir à ce que lui apporte le parent quant aux pratiques qu'il développe avec cet enfant), mais développant une compétence sur les dimensions du traitement du collectif, de la relation avec les parents, les autres professionnelles avec lesquelles il lui faut articuler son action. La qualité est là, faire que le collectif n'annihile pas les singularités, ce qui renvoie à la prise en compte du point de vue de l'enfant et des parents.

\section{Refuser la séparation, l'opposition entre savoir profane et savoir savant}

Une autre voie qui n'est pas incompatible avec la précédente consiste à refuser la séparation entre savoir savant et savoir profane, à récuser l'idée d'une vérité (portée par les professionnelles) en matière de care et d'éducation des jeunes enfants. Il s'agit alors de ne pas considérer que seuls importent les savoirs professionnels liés aux données supposées scientifiques, mais de prendre en compte les traditions et les savoirs d'expériences, en particulier parentaux.

Si nous reprenons le cas des crèches parentales (Brougère, Moreau, 2014), elles accordent plus ou moins aux parents, mais accorder la maximum (ce qui se trouve dans certains cas), implique de refuser de la part du professionnel la posture de vérité, de bonne pratique dans l'absolu. Relativité cela signifie que la pratique (bonne) est située, liée à un contexte, un enfant. Ce qui est bon pour un enfant peut ne pas l'être pour un autre. Cela conduit à une individualisation de la pratique qui ne peut que s'appuyer 
sur les savoirs des parents, savoirs d'expérience concernant leur enfant. Ainsi dans les cas de l'endormissement, du portage, des repas, les professionnelles ont pu remettent en cause la vision de la bonne pratique apprise à l'école au profit d'une attention à la singularité et à la parole des parents concernant leur enfant.

Mais cet exemple est limité et atypique en France où l'on voit plutôt une volonté de rupture entre accueil professionnalisé et garde familiale. Il est très souvent question de marquer la différence en particulier pour éviter une supposée concurrence affective. Cela conduit à développer un geste professionnel qui se distinguerait du geste « amateur ».

Cela exerce une énorme pression sur les personnels qui doivent éviter toute action spontanée, toute marque affective trop importante. Ainsi cette rupture entre geste parental et geste professionnel se paie par une froideur, une distance dont il n'est pas certain qu'elle soit bonne pour l'enfant. A vouloir distinguer le geste professionnel ne met-on pas à distance tout le savoir traditionnel des relations entre adultes et enfants ? C'est sans doute une importance forte donnée à un savoir psychanalytique qui explique la situation française.

Il convient donc d'accepter l'idée de continuité entre expérience parentale et expérience professionnelle, admettre que les parents ont les moyens d'intervenir et de participer, que leur parole doit être écoutée et pas seulement pour leur propre enfant. Cette vision d'une autre dimension de la professionnalité et le refus de la rupture entre savoir scientifique et profane renvoie à une autre façon d'envisager l'apprentissage et la connaissance.

L'expérience parentale génère des connaissances qui sont l'objet le plus souvent d'apprentissages dits informels ${ }^{3}$ : par la pratique, par les échanges entre parents, par la transmission générationnelle, les médias. Sont ainsi générés des répertoires de pratiques hybrides qui peuvent articuler la vulgarisation de savoirs scientifiques (média), des traditions familiales parfois anciennes (mais pas toujours): observation, imitation, participation périphérique, participation guidée sont les modalités principales de ce type d'apprentissage (Brougère, 2011).

Les professionnelles peuvent avoir (au moins partiellement) les mêmes expériences et donc les mêmes apprentissages. Ce que savent et savent faire les professionnelles n'est pas uniquement lié à leurs études ou leur expérience professionnelle. Elles mobilisent également une expérience de parent ou de membre d'une société qui partage certaines caractéristiques culturelles concernant les enfants et leur éducation.

Il faut accepter l'idée que les apprentissages des parents peuvent être réinvestis (n'ont pas vocation à demeurer dans la sphère privée) en particulier dans les structures collectives. En acceptant les enfants on doit accepter ce qui va avec, un cadre éducatif, des pratiques qui entrent avec les enfants, mais aussi les parents pour autant que l'on accepte leur présence, voire leur participation, ce qui est souhaitable.

Nombre d'apprentissages se font dans le cadre de l'activité professionnelle. Les piliers de la professionnalité que nous avons évoqués ne sont pas toujours l'objet d'enseignement et sont donc en partie le résultat d'apprentissage sur le tas, lors des situations de travail. Dans les relations avec les parents et les autres professionnelles on apprend à gérer cette dimension. La dimension collective de

\footnotetext{
${ }^{3}$ Sur cette notion on se reportera entre autres à Brougère (2016).
} 
l'accueil est sans doute davantage l'objet d'enseignement dans les écoles, mais suppose la rencontre sur le terrain et les difficultés qui peuvent en résulter pour être mieux saisie. Il importe de prendre en compte cette dimension essentielle de l'apprentissage en situation de travail, face à la complexité de situations réelles d'accueil des enfants et de leurs parents.

L'enjeu n'est donc pas seulement de développer des formations qui tiennent compte de ces dimensions, mais de reconnaître et supporter les apprentissages en situation informelle. Il faut donc savoir relativiser les apprentissages formels issus de la fréquentation des écoles, non pour les refuser, mais en faire plutôt un point d'appui pour développer des pratiques qui doivent s'enraciner dans des situations concrètes, sans être considérés comme des vérités absolues.

Il y a là une logique globale : reconnaître la valeur des apprentissages en situation informelle (des parents et des professionnelles dans leur expérience privée) et penser qu'une partie de la professionnalisation doit suivre la même voie, se faire en situation. Nous pouvons donc en tirer les conséquences suivantes :

$\checkmark$ ne pas opposer savoirs des professionnelles et savoir des parents : une partie est commune et renvoie à l'enracinement culturel de l'éducation du jeune enfant ;

$\checkmark$ considérer que les connaissances mobilisées pour l'éducation du jeune enfant sont en grande partie situées (liées au lieu, à la culture, la singularité de chaque enfant) ;

$\checkmark$ prendre en compte que les pratiques (les répertoires de pratiques) se construisent in situ et renvoient à diverses sources (la formation et les expériences).

La professionnalisation est en partie un processus d'apprentissage informel : dans l'activité, la relation avec les enfants, les parents, les professionnelles. Ces différents acteurs (sans oublier les enfants) sont des ressources pour les apprentissages. Il faut savoir entendre les enfants, les parents et les autres professionnelles.

Ce processus, souvent implicite, doit être soutenu pour devenir, au moins en partie, explicite dans le cadre d'une pratique réflexive. Il s'agit alors non pas d'appliquer un savoir préexistant mais de construire in situ une pratique partagée et réfléchie. Celle-ci reste ouverte et diverse, en se singularisant selon les enfants et les professionnelles, il n'est pas nécessaire que les professionnelles agissent toutes de la même façon sous réserve de maintenir la coordination et de s'engager dans une coopération et des échanges sur les pratiques.

Il en résulte une vision du préscolaire qui ne se réduit pas à un face à face entre des professionnelles et des enfants, mais un collectif, une communauté de professionnelles, d'enfants et de parents où chacun participe à la construction du répertoire des pratiques partagées. Cette notion empruntée à Barbara Rogoff (2007) renvoie à l'idée que les pratiques sont hétérogènes, issues d'apprentissages divers et constituent des ressources culturelles pour l'action.

Derrière cette vision on trouve l'idée (ou l'idéal) de Wenger (2005) d'une communauté de pratique avec une entreprise commune, un engagement mutuel et un répertoire de pratiques partagées. Chacun y apprend des autres (et pas seulement les enfants). La première rupture est celle-ci, un lieu d'apprentissage qui concerne tous les acteurs et pas seulement les enfants (Brougère, Moreau, 2014). Le terme clé est celui de participation, des professionnelles, des parents et des enfants, comme support de l'apprentissage. Ce qui suppose engagement et affordance : en effet, s'il importe que les acteurs, professionnelles et enfants comme parents puissent s'engager dans la structure, il est tout aussi important que celle-ci offre des possibilités d'action, de participation aux différents acteurs et en particulier aux parents, ce que l'on peut appeler les affordances (Billett, 2004). Donner la possibilité à 
chacun de participer est essentiel pour l'enrichissement des pratiques et l'apprentissage. Une structure d'accueil préscolaire doit être pensée comme un espace qui permette à tous, aux enfants, aux professionnelles et aux parents de participer à cette entreprise commune. Les apprentissages divers en résulteront nécessairement. Il importe donc de penser ces lieux comme des lieux où il est possible pour chacun de faire, de participer et sans doute au-delà de découvrir, d'explorer (de nouvelles façons de faire, de nouveaux objets, de nouveaux lieux, de nouvelles situations). Ce que nous valorisons c'est une symétrie généralisée, une logique qui vaut pour tous, enfants comme adultes, et qui permettent à tous d'apprendre.

\section{Références bibliographiques}

Billett, S. (2004). Working participatory practices: conceptualising workplaces as learning environments. Journal of Workplace Learning, vol. 16, $\mathrm{n}^{\circ}$ 5/6, pp.312-324.

Brougère G. (2011), «Apprendre en participant». In E. Bourgeois, G. Chapelle, Apprendre et faire apprendre, Nouvelle édition, Paris : PUF, pp. 115-124.

Brougère G. (2016), « De l'apprentissage diffus ou informel à l'éducation diffuse ou informelle », Le Télémaque, ${ }^{\circ} 49$, pp. 51-64.

Brougère G., Moreau A. (2014) «Participation parentale, pratiques partagées et diversité ». In S. Rayna \& G. Brougère (dir.) Petites enfances, migrations et diversités, Bruxelles : P.I.E. Peter Lang, pp. 137-163.

Brougère G., Roucous L. et Chanu L. (2001) «Des assistantes maternelles à la Ludothèque : du jeu dans la professionnalisation », Recherches et Prévisions, $\mathrm{n}^{\circ}$ 64, pp. 35-47.

Rogoff, B. et al. (2007). «Développement des répertoires culturels et participation des enfants aux pratiques quotidiennes ». In G. Brougère \& M. Vandenbroeck (dir.), Repenser l'éducation des jeunes enfants. Bruxelles : P.I.E. Peter Lang, pp.103-138.

Rupin P. (2014), Participation et apprentissages d'adultes en milieu préscolaire communautaire. L'exemple du Chili, Thèse de doctorat, Université Paris 13.

Tronto, J. (2009). Un monde vulnérable. Pour une politique du care, Paris : La Découverte.

Wenger, E. (2005). La théorie des communautés de pratique. Apprentissage, sens et identité. Sainte Foy : Presses de l'Université Laval. 